\title{
NEW APTES CROSS-LINKED POLYMERS FROM \\ POLY(ETHYLENE OXIDE)S AND CYANURIC CHLORIDE FOR LITHIUM BATTERIES
}

\author{
Dean M. Tigelaar, ${ }^{* \dagger}$ Mary Ann B. Meador, James D. Kinder, William R. Bennett ${ }^{\ddagger}$ \\ NASA Glenn Research Center, 21000 Brookpark Rd., Cleveland, OH 44135
}

\begin{abstract}
* To whom correspondence should be addressed. E-mail: dean.m.tigelaar@grc.nasa.gov.
$\dagger$ Employed by Ohio Aerospace Institute.

\$ Employed by QSS Group, Inc.
\end{abstract}

\begin{abstract}
A new series of polymer electrolytes for use as membranes for lithium batteries are described. Electrolytes were made by polymerization between cyanuric chloride and diamino-terminated poly(ethylene oxide)s, followed by cross-linking via a sol-gel process. Thermal analysis and lithium conductivity of freestanding polymer films were studied. The effects of several variables on conductivity were investigated, such as length of backbone PEO chain, length of branching PEO chain, extent of branching, extent of cross-linking, salt content, and salt counterion. Polymer films with the highest percentage of PEO were found to be the most conductive, with a maximum lithium conductivity of $3.9 \times 10^{-5} \mathrm{~S} / \mathrm{cm}$ at $25^{\circ} \mathrm{C}$. Addition of plasticizer to the dry polymers increased conductivity by an order of magnitude.

This is a preprint of an article submitted to a journal for publication. Since revisions may be made prior to formal publication, this version is made available with the understanding that it will not be cited or reproduced without the permission of the author.
\end{abstract}




\section{Introduction}

Polymer electrolytes offer advantages in lithium batteries, such as design flexibility and the ease of polymer processing compared to solid crystalline and glassy materials. ${ }^{1}$ However, low room temperature lithium ion conductivities of solid polymer electrolytes still hinders their use in commercial applications. For more demanding space applications such as the Mars rovers, astronaut suits, and manned surface vehicles, acceptable performance is desired down to $-40^{\circ} \mathrm{C}$. Most polymeric materials that have been studied are based on poly(ethylene oxide) (PEO) because of their strong solvation of lithium ions. ${ }^{2}$ However, at temperatures below $80^{\circ} \mathrm{C}$, PEO films tend to crystallize, dramatically decreasing conductivity. ${ }^{3-4}$ Furthermore, for many battery applications even high molecular weight PEO has unacceptable mechanical properties, especially at higher temperatures.

Our laboratory has recently reported a series of rod-coil polymers for lithium batteries that display dimensionally stable films with good ionic conductivity. ${ }^{5}$ The polymer films are completely amorphous at room temperature when doped with lithium trifluoromethane sulfonimide. The rod segments consist of rigid linear or branched polyimides and the coil segments are oligomeric alkylene oxide chains. It has been proposed that good mechanical and transport properties, as well as the amorphous nature of the alkylene oxide chains, are due to phase separation between the rod and coil segments. It was also observed that increased branching and molecular weight lead to increased conductivity and dimensional stability.

Herein we discuss the properties of copolymers made from inorganic siloxane and polyalkylene oxide units connected by melamine linkages (see Scheme 1). Melamine is 
chosen as the linking unit because it provides a branching site, cation binding sites to help ionic transport between polymer chains, and the opportunity for self assembly through hydrogen bonding. In addition, the polymers are easily made by the reaction of cyanuric chloride with a series of amine-terminated alkylene oxides (Jeffamines ${ }^{\circledR}$, Huntsman Corporation shown in Scheme 2). A linear polymer is first made by reacting two of the aryl chlorides with the diamino alkylene oxides (Scheme 3), followed by reaction of the third site on cyanuric chloride with varying ratios of monoamino-alkylene oxides and (3aminopropyl)triethoxysilane (APTES). The APTES provides a cross-linking unit to increase the mechanical strength of the resulting film. The lithium trifluoromethane sulfonimide-doped polymers are cross-linked through a sol-gel process to form freestanding films. ${ }^{6-8}$ Several parameters have been varied in this study, including the oxygen to lithium ratio, molecular weight of the alkylene oxide diamine oligomers used in the backbone (MW=600, 2000), molecular weight of the monoamine side chain oligomers (MW=1000, 2620), and ratio of alkylene oxide oligomer to APTES in the side chain to understand the effect of these variables on the ionic conductivity and other properties of the resulting polymer films.

\section{Experimental}

Materials. Cyanuric chloride, and (3-aminopropyl)triethoxysilane (APTES) were purchased from Aldrich Chemical Company and used as received. Diisopropylethylamine was purchased from Aldrich in Biotech grade and used as received. Lithium bis(trifluoromethane)sulfonimide was purchased from Aldrich in $99.95 \%$ purity, dried at $140^{\circ} \mathrm{C}$ under vacuum for 10 hours, and stored in a dry box. 
Monoamine and diamine-capped polyalkylene oxide oligomers (Jeffamine XTJ-500, XTJ-502, XTJ-234, and XTJ-506) were obtained from Huntsman Corporation. All glassware was oven-dried for at least 24 hours prior to use. Tetrahydrofuran (THF) was distilled from sodium/benzophenone prior to use.

Instrumentation. Polymers were characterized by differential scanning calorimetry (DSC) from -90 to $300^{\circ} \mathrm{C}$ using a Q1000 calorimeter manufactured by TA instruments. Thermal gravimetric analysis (TGA) was obtained using a TA instruments high resolution TGA2950 analyzer. ATR-FTIR spectra of polymer films were taken with a Nicolet Nexus 470 FTIR spectrometer equipped with a Smart Omni sample ATR accessory. Solid ${ }^{13} \mathrm{C}$ NMR spectra of the polymer films were obtained on a Bruker Avance 300 Spectrometer using cross-polarization and magic angle spinning at $7 \mathrm{kHz}$. The acquisition also employed spinning sideband suppression using a CPSELTICS sequence. The solid ${ }^{13} \mathrm{C}$ spectra were externally referenced to the carbonyl of glycine (176.1 ppm relative to tetramethylsilane, TMS).

Ionic conductivity was measured using electrochemical impedance techniques, using a Solartron/Schlumberger model 1250 FRA and model 1286 Electrochemical Interface. ${ }^{5}$ Conductivity was measured over the range of 0 to $80^{\circ} \mathrm{C}$.

Experimental analysis was carried out using RS/Series for Windows, including RS/1 version 6.01, and RS/Discover and RS/Explore Release 4.1, available from Domain Manufacturing Corporation.

Polymer Synthesis. A typical synthesis of the polymer is given for a formulation containing Jeffamine diamine of MW 2000 in the linear backbone and 50\% APTES, 50\% Jeffamine (MW 2620) on the branching site (run 40 from Table 1). 
XTJ-502 (13.40 g, $6.70 \mathrm{mmol})$ and diisopropylethylamine $(3.6 \mathrm{~mL}, 21 \mathrm{mmol})$ were dissolved in $30 \mathrm{~mL}$ THF. The reaction temperature was lowered to $0^{\circ} \mathrm{C}$ in an ice bath. Cyanuric chloride $(1.24 \mathrm{~g}, 6.70 \mathrm{mmol})$ dissolved in $2 \mathrm{~mL}$ THF was then added to the solution. The solution immediately changed color from clear to yellow. The reaction was slowly warmed to room temperature and stirred overnight. The resulting viscous solution was filtered to remove a crystalline white solid (protonated base) that had formed during the course of the reaction. The filter cake was washed with THF and the resulting filtrate was concentrated to the original reaction volume by rotary evaporation. The 2620 molecular weight monoamine, XTJ-234 (8.84 g, $3.37 \mathrm{mmol})$, and APTES $(0.753 \mathrm{~g}, 3.40 \mathrm{mmol})$ were added to the filtrate and the reaction was heated to $130^{\circ} \mathrm{C}$ overnight. The resulting cloudy solution was then weighed and determined to be $55.2 \%$ polymer by weight assuming $100 \%$ yield.

Lithium trifluoromethane sulfonimide $(0.711 \mathrm{~g}$, corresponding to a $21: 1 \mathrm{O}$ to $\mathrm{Li}$ ratio) was then dissolved in a $4.55 \mathrm{~g}$ aliquot (2.51 $\mathrm{g}$ of polymer) of this solution. The solution was poured into a $60 \mathrm{~mm}$ diameter Teflon dish and air-dried in a fume hood for 24 hours. The resulting sticky solid was then dried under vacuum at room temperature to $80^{\circ} \mathrm{C}$ until bubbling within the film ceased, followed by further curing at $160^{\circ} \mathrm{C}$ under vacuum overnight. The film was immediately transferred into a dry box, cooled, removed from the Teflon dish, and sealed in an air-tight bag. Films that were made in entries 1, 2, 37, and 38 in Table 1 were too sticky to remove from the Teflon dish and were cast into aluminum pans instead. Lithium conductivities of these films were measured while attached to the aluminum backing. 


\section{Results and discussion}

Polymer synthesis and characterization. The reaction of cyanuric chloride with various alkyl and aromatic amines has been reported previously. ${ }^{9}$ Simanek and coworkers have recently reported the synthesis of dendrimers in which melamine is the

core unit. ${ }^{10-12}$ They also demonstrated the chemoselective reactivity of the three reactive sites on cyanuric chloride at various temperatures. Reaction of one site takes place at $0^{\circ} \mathrm{C}$, the second site at room temperature, and the third site above $70^{\circ} \mathrm{C}$. Jan and coworkers have used similar methodology to synthesize linear poly(alkylene oxide)s by the reaction of various Jeffamines with two of the reactive sites of cyanuric chloride in the presence of base. ${ }^{13}$ Through gel permeation chromatography analysis, they found $\mathrm{M}_{\mathrm{w}}$ 's of 38,000 and 28,700 using Jeffamine D-2000 (poly(propylene oxide) (PPO)) and Jeffamine D-2001 (PEO), respectively. Unfortunately, even at relatively high molecular weights, they report that these polymers exist as viscous liquids and semi-solids. We show that through the addition of APTES to the third reactive site, the polymers become cross-linked and behave as elastomeric solids.

Scheme 2 shows the synthesis of lithium bis(trifluoromethane)sulfonimide-doped PEO's that can be cast into freestanding films. First, the linear polymer was made by the reaction of equimolar amounts of cyanuric chloride with diamines XTJ-500 (MW=600) or XTJ-502 $(\mathrm{MW}=2000)$ in THF at room temperature. These diamines contain a mixture of ethylene oxide and propylene oxide units in the chain. The ethylene oxide promotes lithium ion conductivity while the propylene oxide units help suppress crystallization. Diisopropylethylamine (Hünig's base) was added to remove $\mathrm{HCl}$ generated in the reaction medium. The ammonium salt precipitates during the reaction and is removed by 
filtration. No gel formation was observed during the reaction, which gives evidence that the polymer is not cross-linking prematurely via reaction of the third reactive site on cyanuric chloride. Once the linear polymer is made, the third site is reacted with monoamines XTJ-506 (MW=1000) or XTJ-234 $(\mathrm{MW}=2620)$ at $130^{\circ} \mathrm{C}$. In addition, APTES in varying amounts is also added to the reaction to provide for a cross-linked network once films are cast. Polymer solutions are doped with lithium salts and cast into films in Teflon dishes. Three different lithium salt concentrations measured by the ratio of PEO oxygen to lithium were evaluated $(21: 1,14: 1,7: 1)$. Films are typically air-dried overnight in a fume hood, followed by drying and further curing under full vacuum at $160^{\circ} \mathrm{C}$ overnight. The resulting films were immediately transferred into a dry box and sealed into air-tight bags to avoid any absorption of water.

The infrared spectrum of polymer 42 exhibits peaks that correspond to the PEO backbone. Peaks were observed at 2887 (CH stretch), $1349\left(\mathrm{CH}_{2}\right.$ scissors $), 1099$, and 1059 (C-O-C asym. stretch) $\mathrm{cm}^{-1}$. Solid ${ }^{13} \mathrm{C}$ NMR was used to characterize the cured polymer films. The most dominant peak in the spectra is a broad resonance at $70.7 \mathrm{ppm}$ that can be assigned to all of the methylene and methyne carbons in the alkylene oxide units in the diamine and monoamine portions of the polymers. The methyl carbons from the propylene oxide units appear at $17.6 \mathrm{ppm}$, while a peak at $47.2 \mathrm{ppm}$ can be assigned to the nitrogen substituted carbons at the termini of the diamines and monoamines. The melamine carbons in the spectra of the fully cured polymers appear as a small broad peak at $163.6 \mathrm{ppm}$, while the APTES carbons appear at 11.3, 21.5 and $47.2 \mathrm{ppm}$ in the polymer. 
The cross-linked polymer films are strong and highly elastic in nature. Figure 1 shows an example of the flexibility of an undoped film that contains 2000 MW Jeffamine diamine in the main polymer chain, and a 1:1 ratio of 2620 Jeffamine monoamine to APTES in the branching site. This film contains the lowest amount of cross-linking and highest conductivity of all freestanding films studied in the doped form. Moreover, the film is strong and flexible enough to be stretched to over seven times its initial length, and completely relax back to its original shape.

A summary of the data for the films studied is given in Table 1, including room temperature conductivity for the lithium containing films, $T_{g}$, and $T_{d}$. TGA data from Table 1 shows that polymer films have an onset of decomposition in excess of $350{ }^{\circ} \mathrm{C}$. DSC analysis shows a strong crystalline transition around room temperature for films that are not doped with lithium salt. However, when lithium salt is added, this transition disappears (Figure 2). This suppression of crystallization leads to improved room temperature lithium conductivity compared to state of the art PEOs. These results are similar to those reported previously from our laboratory in rod-coil polymers that contain Jeffamines $^{5}$ giving evidence that having small amounts of propylene oxide in the mono and diamines may play a critical role in keeping the films completely amorphous down to below room temperature. As is the case in most other solid polymer electrolytes, $T_{g}$ values tend to increase with an increase in salt concentration. In addition, lithium conductivity tends to decrease with increasing polymer $\mathrm{T}_{\mathrm{g}}$. The most conductive samples have $T_{\mathrm{g}}$ between -40 and $-60^{\circ} \mathrm{C}$. Less conductive samples have $\mathrm{T}_{\mathrm{g}}$ 's that are higher than $-40^{\circ} \mathrm{C}$. 
The salt containing films from Table 1 can be considered as an experimental design in four variables: diamine type ( $d$, molecular weights of 600 and $2000 \mathrm{~g} / \mathrm{mole})$, monoamine type ( $m$, molecular weights of 1000 and $2620 \mathrm{~g} / \mathrm{mole}$ ), percent APTES replacing the monoamine in the third reactive site $(s, 25$ to $75 \%)$ and $\mathrm{O}$ to $\mathrm{Li}$ ratio $(l, 7$ to 1,14 to 1 or 21 to 1). Diamine type and monoamine type are discrete (non-numerical) variables evaluated at two levels while salt concentration and \% APTES are continuous (numerical) variables evaluated at 3 or 4 levels each. This allows an empirical model to be derived to describe the relationship of room temperature ionic conductivity to each of the variables by entertaining a quadratic model of the form :

Response (log conductivity) $=\mathrm{A}+\mathrm{B} * m+\mathrm{C} * d+\mathrm{D} * s+\mathrm{E} * l+\mathrm{F}^{*} s^{2}+\mathrm{G} * l^{2}+\mathrm{H}^{*} m * d+$ $\mathrm{I} * m * s+\mathrm{J} * m * l+\mathrm{K} * d * s+\mathrm{L} * d * l+\mathrm{N} * s * l$

where $m$ is monoamine molecular weight, $d$ is diamine molecular weight, $s$ is \% APTES and $l$ is $\mathrm{O}: \mathrm{Li}$ ratio and $\mathrm{A}$ through $\mathrm{N}$ are coefficients empirically derived from modeling the experimental data. The full model contains first order effects of all four variables and second order terms for $\mathrm{s}$ and $\mathrm{l}$, as well as all possible two-way interaction terms. (Owing to the discrete nature of variables monoamine type and diamine type, there is no physical meaning to a second order term in these variables.)

Values for the room temperature ionic conductivity shown in Table 1 were analyzed using linear least squares regression. The conductivity was normalized using a $\log$ transform before modeling. All independent variables were orthogonalized (transformed to the -1 to 1 range) prior to modeling. Terms not deemed to be statistically significant 
( $<90 \%$ confidence) were dropped from the model one at a time using a stepwise modeling technique. In this case, significant terms include all main effects and three interactive/synergistic terms, $d^{*} m, m^{*} s$ and $d^{*} s$. All terms in the model were greater then $98.5 \%$ significant. Standard error of the regression was 0.071 with an $\mathrm{R}^{2}=0.98$.

A three dimensional graph of the resulting response surface model for log ionic conductivity plotted versus lithium to oxygen ratio and APTES percent is shown in Figure 3. Four separate surfaces are shown for the four combinations of monoamine and diamine used in the formulations. Overall, the films containing 2000 MW diamine and 2620 MW monoamine (green surface) have the highest conductivities, followed by those made with $2000 \mathrm{MW}$ diamine and $1000 \mathrm{MW}$ diamine (blue surface), and those with 600 MW diamine and $2620 \mathrm{MW}$ monoamine (yellow surface). The films made with $600 \mathrm{MW}$ diamine and $1000 \mathrm{MW}$ monoamine (red surface) have the lowest overall conductivities.

The synergistic/interactive effect of diamine with monoamine $\mathrm{MW}\left(d^{*} m\right)$ is illustrated by the surfaces in Figure 3. Comparing the red surface to the blue surface (both representing films containing the $1000 \mathrm{MW}$ monoamine) and the green surface to the yellow surface (both representing films containing the 2620 MW monoamine), it is evident that films having the $2000 \mathrm{MW}$ diamine in the polymer backbone have as much as a 10 -fold increase in conductivity over films with the $600 \mathrm{MW}$ diamine in the polymer backbone. This trend can not only be explained by the fact that films containing 2000 MW diamine have longer chains of alkylene oxides within the films and therefore lower $\mathrm{T}_{\mathrm{g}}$, but the $2000 \mathrm{MW}$ diamine also has a higher percentage of ethylene oxide to propylene oxide than the $600 \mathrm{MW}$ diamine ( 7.9:1 vs. $\sim 2.5: 1)$. Propylene oxide is a poorer conductor of lithium. 
By comparing the blue surface to the green surface (both representing films containing the $2000 \mathrm{MW}$ diamine) and the red surface to the yellow surface (both representing films containing the $600 \mathrm{MW}$ diamine), it is evident that increasing the MW of the monoamine side chain from 1000 to 2620 increases the conductivity, although the effect is larger for the $600 \mathrm{MW}$ diamine. For polymers with a $600 \mathrm{MW}$ backbone, there is as much as a seven fold increase in conductivity. For films that contain $2000 \mathrm{MW}$ PEO in the polymer backbone, increasing the MW of the PEO branch from 1000 to 2620 has a less dramatic effect on the conductivity. In this case, the conductivity is increased by at most a factor of two.

The synergistic/interactive effects of both monoamine and diamine type with percent APTES ( $m *_{s}$ and $d^{*} s$, respectively) is also illustrated by the surfaces shown in Figure 3. Within each surface (having the same diamine and monoamine combination), conductivity decreases with the amount of cross-linking APTES added. This effect is most dramatic for the films containing $600 \mathrm{MW}$ diamine and $1000 \mathrm{MW}$ monoamine (red surface) which shows nearly a 10 fold change in conductivity over the range of percent APTES modeled. For polymers with the 600 MW diamine and 2620 diamine (yellow surface), there is only a two-fold change in conductivity over the same range For films made with $2000 \mathrm{MW}$ diamine (blue and green), the decrease in conductivity with increasing APTES content is very small.

For all surfaces, conductivity decreases as the amount of lithium relative to oxygen is increased. Furthermore, since there is no interactive term with salt in the model, this relationship is the same for all combinations of diamine and monoamine used. Increasing 
the lithium to oxygen ratio also had an adverse effect on the mechanical properties of the polymer films. The films become softer and more sticky.

The least conductive (600 MW diamine and $1000 \mathrm{MW}$ monoamine) series has the largest drop-off in ionic conductivity as temperature is decreased as shown in Figure 4 for formulations containing 50\% APTES and 21:1 O to Li. This is most likely because polymers with shorter PEO chains require an increase in temperature to activate the transport of lithium between chains. The other three formulations show nearly identical trends with temperature.

The values for $\mathrm{T}_{\mathrm{g}}$ from Table 1 were also modeled using multilinear regression (Figure 5). Significant terms for the $T_{g}$ model include all main effects and three interactive terms, $d^{*} m, m^{*} l$ and $d^{*} s$. All terms in the model were greater then $98.3 \%$ significant. Standard error of the regression was $2.1{ }^{\circ} \mathrm{C}$ with an $\mathrm{R}^{2}=0.87$. It is easiest to view the surfaces as two distinct pairs, those with the $600 \mathrm{~g} / \mathrm{mole}$ diamine in the backbone and those with the $2000 \mathrm{~g} / \mathrm{mole}$ diamine in the backbone. Those with 600 MW diamine in the backbone show a large increase in $T_{g}$ with increasing silane concentration, and those with $2000 \mathrm{MW}$ diamine in the backbone show a much flatter response to silane concentration.

In general, the $\mathrm{T}_{\mathrm{g}}$ is lower in films that contain the longer monoamine ( $\mathrm{MW}=$ $2620 \mathrm{~g} / \mathrm{mole}$ ) as a sidechain. At $-54{ }^{\circ} \mathrm{C}$, the film that has the lowest $\mathrm{T}_{\mathrm{g}}$ is that made from $600 \mathrm{~g} /$ mole diamine in the backbone and 75\% $2620 \mathrm{MW}$ monoamine (25\% APTES) with 21 to $1 \mathrm{O}$ to $\mathrm{Li}$ ratio. This is probably because with the $600 \mathrm{~g} / \mathrm{mole}$ diamine in the backbone, there is a higher frequency of branching sites along the backbone, leading to a lower $T_{\mathrm{g}}$. However, this film is not as conductive as the film with $2000 \mathrm{~g} / \mathrm{mole}$ diamine 
and $75 \% 2620 \mathrm{~g} /$ mole monoamine with the same salt concentration $\left(\mathrm{T}_{\mathrm{g}}=-51.4{ }^{\circ} \mathrm{C}\right)$. This is most likely due to the $600 \mathrm{~g} /$ mole diamine having a higher percentage of PPO than the 2000 MW backbone, which counteracts the improved freedom of movement.

Two samples were tested for conductivity in which no lithium salt was added (entries 33 and 36 in Table 2). These samples both had ionic conductivities of about $1 \times 10^{-8} \mathrm{~S} / \mathrm{cm}$, or three orders of magnitude less than the lithium-doped samples. The ammonium salt that is formed by protonation of diisopropylethylamine during the course of the reaction precipitates and is removed by filtration after the reaction with the second site on cyanuric chloride. The reaction had to be filtered at this point because the precipitate disappears after reaction with APTES. The conductivity data suggests that any remaining dissolved ammonium salt is subsequently incorporated into the polymer film, although it has a minor effect on conductivity. If needed, the residual ammonium salt can be removed by filtering the polymer solution through basic alumina prior addition of lithium salt. The alumina is then washed with THF until the filtrate is no longer UV active.

Effects of salt and plasticizer. A brief study was performed to observe the effects of adding propylene carbonate (PC) as a plasticizer to these polymers. Kim and Smotkin have shown previously that the addition of $10 \%$ PC to high MW PEO films doped with lithium trifluoromethanesulfonimide increases lithium conductivity by a factor of two between 50 and $80^{\circ} \mathrm{C} .{ }^{14}$ A polymer having $2000 \mathrm{MW}$ diamine, $2620 \mathrm{MW}$ monoamine and 50\% APTES (same formulation as entry 40 in Table 1) was chosen for the study. Formulations with $10,20,40$, and $80 \mathrm{wt} \%$ PC were added to the film after curing.

It should be noted that in films that contained $20 \%$ to $80 \%$ PC, there was a significant decrease in conductivity after the initial heating cycle during conductivity 
measurements. This drop in conductivity appeared to be proportional to the initial amount of PC added, which would give evidence that a portion of the PC evaporates during heating. This is shown in Figure 6 for the $80 \%$ PC sample. Films were heated from room temperature to $80^{\circ} \mathrm{C}$, with a measurement every $20^{\circ} \mathrm{C}$. The sample was then cooled and measured at the same temperatures. Room temperature conductivities summarized in Table 2 are those measured after the heating cycle only. Similar to the previously reported results with PEO, adding $10 \%$ PC doubles the conductivity as shown in Table 2. When PC is increased to $80 \%$, the conductivity is increased by an order of magnitude. However, unlike PEO, even with as much as $80 \%$ PC added to the polymer film, the film maintains excellent mechanical strength. The films do swell after the addition of PC, from an initial diameter of $62 \mathrm{~mm}$ to 63,64 , and $75 \mathrm{~mm}$ for films in which 20,40 , and $80 \%$ PC was added, respectively. Glass transitions were not observed down to $-90{ }^{\circ} \mathrm{C}$ for any of the films in which $\mathrm{PC}$ was added.

Experiments were also run in which lithium trifluoromethanesulfonimide was replaced with lithium perchlorate and lithium trifluoromethane sulfonate. Films that were made with the latter two salts were slightly less conductive. Surprisingly, these two salts also had adverse effects on the film's mechanical properties. These films are much more sticky compared to the parent films doped with lithium trifluoromethanesulfonimide, even though films doped with lithium trifluoromethanesulfonimide are expected to have less mechanical strength since the trifluoromethanesulfonimide counterion is known to have a plasticizing effect on polymer films. ${ }^{15}$ 


\section{Conclusions}

This paper describes a new alternating copolymer system that has been synthesized for use as a solid electrolyte for lithium batteries. The polymer is made by the reaction of diamino-terminated alkylene oxides with cyanuric chloride, followed by reaction with monoamino-terminated alkylene oxides and (3-aminopropyl)triethoxysilane (APTES). The latter reagent serves of the cross-linking agent to provide dimensional stability to the polymer film.

A systematic study was performed, in which oxygen to lithium ratio, molecular weight of the PEO backbone (MW=600, 2000), molecular weight of the side chains (MW=1000, 2620), and percent APTES as cross-linker were varied. Films were characterized using AC impedance to measure lithium conductivity, TGA, and DSC. Films that were found to be the most conductive contained a 21:1 oxygen:lithium ratio, a $2000 \mathrm{~g} / \mathrm{mole}$ Jeffamine diamine in the polymer backbone, $75 \% 2620 \mathrm{~g} /$ mole Jeffamine monoamine and 25\% APTES in the side chain, and lithium trifluoromethanesulfonimide as the lithium salt. The conductivity at $25^{\circ} \mathrm{C}$ for this film is $3.9 \times 10^{-5} \mathrm{~S} / \mathrm{cm}$. Lithium conductivity decreases with increasing amounts of cross-linking agent but film strength increases. Slightly higher conductivities may be obtained with polymers having less than 25 percent APTES cross-linker. However, dimensional stability will be compromised as APTES percent approaches zero. In fact, films with $2000 \mathrm{~g} / \mathrm{mole}$ Jeffamine diamine in the backbone that contained less than 50\% APTES were too weak to be produced as freestanding films.

One polymer system with nearly as high room temperature conductivity but much better mechanical integrity $(2000 \mathrm{~g} /$ mole Jeffamine diamine in the backbone, $50 \% 2620$ $\mathrm{g} /$ mole Jeffamine monoamine and 50\% APTES in the side chain, and 21:1 O:Li) was 
chosen for further study. In these films, the lithium counterion was varied and propylene carbonate (PC) was added as a plasticizer. Films were found to have both the highest conductivity and best dimensional stability when trifluoromethanesulfonimide was chosen as the counterion. Addition of $20-80 \mathrm{wt} \%$ PC increased lithium conductivity by almost an order of magnitude.

Acknowledgements. We thank Daniel Scheiman for DSC and TGA analysis. We thank Carolyn Youngman for lithium conductivity analysis. We thank the Polymer Rechargeable Energy Systems Program (PERS) of NASA Glenn Research Center for support of this work. D. M. T. was supported by NASA cooperative agreement NCC31089.

\section{References}

1. Gray, F. M. Polymer Electrolytes, Springer-Verlag: New York, 1997.

2. Polymer Electrolyte Review, Vol. 1, ed. MacCallum, J. R., Vincent, C. A., Elsevier, Amsterdam, 1987.

3. Fu, Y.; Pathmanathan, K.; Stevens, J. R., J. Chem. Phys., 1991, 94, 6323.

4. Chung, S. H.; Jeffery, K. R.; Stevens, J. R., J. Chem. Phys., 1991, 94, 1803

5. a) Meador, M. A. B.; Cubon, V. A.; Scheiman, D. A.; Bennett, W. R. Chem. Mater. 2003, 15, 3018. b) "Preliminary Evaluations of Polymer-based Lithium Battery Electrolytes under development for the Polymer Electrolyte Rechargeable Systems Program", William R. Bennett, Michelle. A. Manzo, 1st International IECEC Conference, Portsmouth, VA. (2003).

6. Judeinstein, P.; Titman, J.; Stamm, M.; Schmidt, H., Chem. Mater. 1994, 6, 127. 
7. Popall, M.; Andrei, M.; Kappel, J.; Kron, J; Olma, K.; Olsowski, B., Electrochem. Acta, 1998, 43, 1155.

8. Bermudez, V.de Zea; Alcacer, L.; Acosta, J. L.; Morales, E., Solid State Ionics, 1999, $116,197$.

9. a) Kaiser, D. W.; Thurston, J. T.; Dudley, J. R.; Schaefer, F. C. Hechenbleikner, I.; Holm-Hansen, D J. Am. Chem. Soc. 1951; 73, 2984. b) Choi, I. S.; Li, X.; Simanek, E. E.; Akaba, R.; Whitesides, G. M.; Chem. Mater. 1999; 11, 684. c) Mathias, J. P.; Simanek, E. E.; Whitesides, G. M.; J. Am. Chem. Soc.; 1994; 116 ; 4326.

10. Zhang, W.; Simanek, E. E. Org. Lett., 2000, 2, 843.

11. Zhang, W.; Nowlan, D. T. III; Thomson, L. M.; Lackowski, W. M.; Simanek, E. E. J. Am. Chem. Soc. 2001, 123, 8914.

12. Steffensen, M. B.; Simanek, E. E. Org. Lett., 2003, 5, 2359.

13. Jan, L. Z.; Huang, B. H.; Lin, J.-J. Polymer, 2003, 44, 1003.

14. Kim, Y-T.; Smotkin, E. S. Solid State Ionics, 2002, 149, 29.

15. Armand, M., Kadiri, F. El., Proc. Symp. Lithium Batteries, ed. Dey, A. N., Vol 87-1, p. 502, Electrochem. Soc.: Pennington, NJ, 1987 


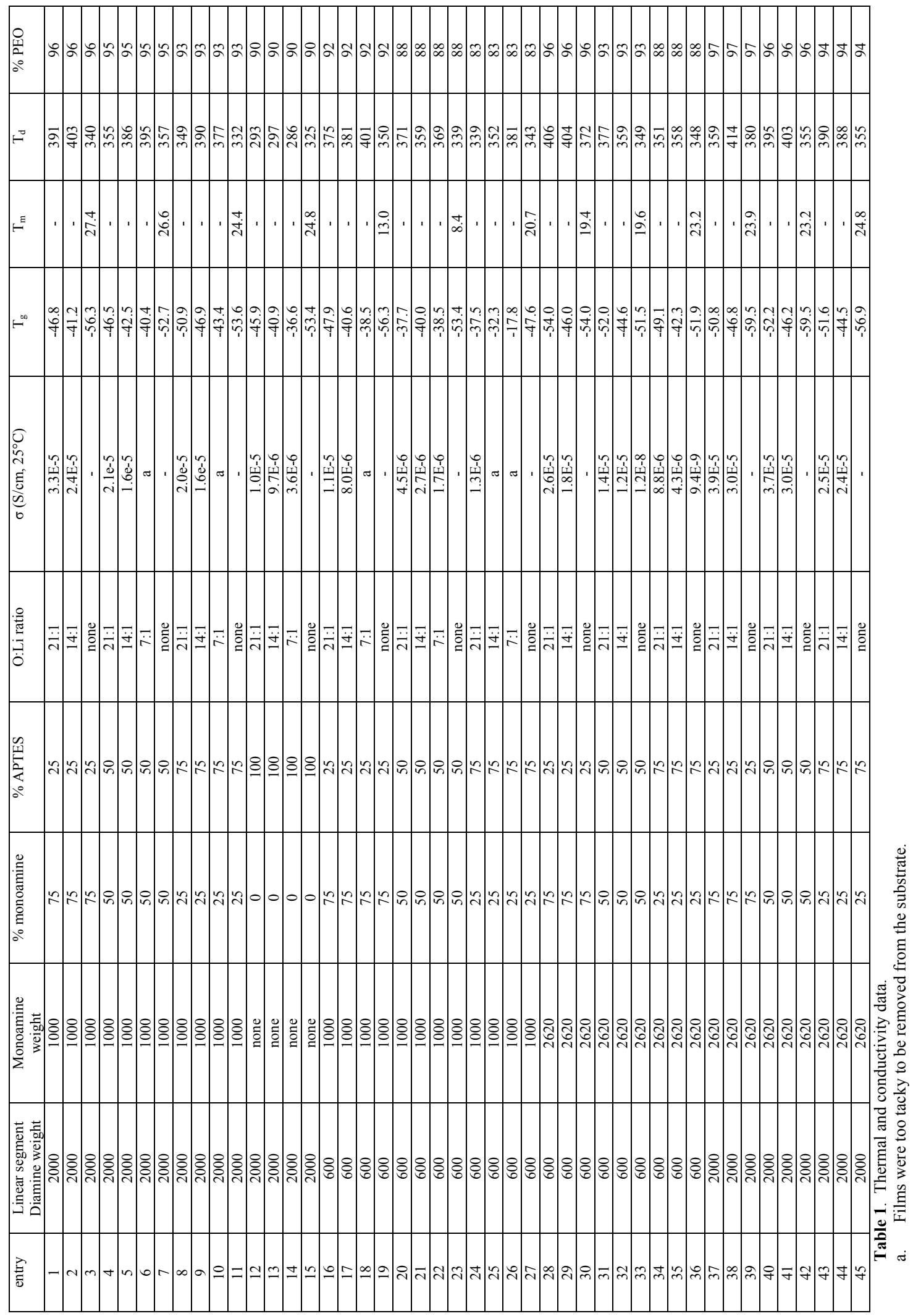




\begin{tabular}{|c|c|c|c|}
\hline Salt & $\begin{array}{c}\text { \% Propylene } \\
\text { carbonate added }\end{array}$ & $\mathrm{T}_{\mathrm{g}}$ & $\sigma\left(\mathrm{S} / \mathrm{cm}, 25^{\circ} \mathrm{C}\right)$ \\
\hline $\mathrm{LiN}\left(\mathrm{SO}_{2} \mathrm{CF}_{3}\right)_{2}$ & none & -52.2 & $3.6 \mathrm{E}-5$ \\
\hline $\mathrm{LiN}\left(\mathrm{SO}_{2} \mathrm{CF}_{3}\right)_{2}$ & 10 & - & $6.8 \mathrm{E}-5$ \\
\hline $\mathrm{LiN}\left(\mathrm{SO}_{2} \mathrm{CF}_{3}\right)_{2}$ & 20 & - & $2.2 \mathrm{E}-4$ \\
\hline $\mathrm{LiN}\left(\mathrm{SO}_{2} \mathrm{CF}_{3}\right)_{2}$ & 40 & - & $2.9 \mathrm{E}-4$ \\
\hline $\mathrm{LiN}\left(\mathrm{SO}_{2} \mathrm{CF}_{3}\right)_{2}$ & 80 & - & $3.1 \mathrm{E}-4$ \\
\hline $\mathrm{LiSO}_{3} \mathrm{CF}_{3}$ & none & -50.0 & $1.7 \mathrm{E}-5$ \\
\hline $\mathrm{LiClO}_{4}$ & none & -49.1 & $1.2 \mathrm{E}-5$ \\
\hline
\end{tabular}

Table 2. Effects of counterion and plasticizer on the $\mathrm{T}_{\mathrm{g}}$ and lithium conductivity of polymer 40. 


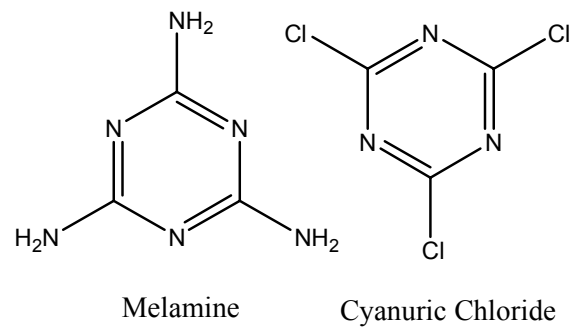

\section{Scheme 1}<smiles>CC(N)COC(C)COCC(C)OCC(C)N</smiles>

$$
\begin{array}{ll}
\text { Jeffamine XTJ-502 } & \text { Jeffamine XTJ-500 } \\
\mathrm{b}=39.5 & \mathrm{~b}=9.0 \\
\mathrm{a}+\mathrm{c}=5 & \mathrm{a}+\mathrm{c}=3.6 \\
\mathrm{MW}=2000 & \mathrm{MW}=600
\end{array}
$$<smiles>[R]C(N)C(C)OC(C)COCCOC</smiles>

$$
\begin{array}{ll}
\text { Jeffamine XTJ-506 } & \text { Jeffamine XTJ-234 } \\
\mathrm{EO}=19 & \mathrm{EO}=49 \\
\mathrm{PO}=3 & \mathrm{PO}=8 \\
\mathrm{MW}=1000 & \mathrm{MW}=2620
\end{array}
$$

Scheme 2. Monoamine and diamine-capped alkylene oxide oligomers that were used along with their approximate molecular weights and ratios of ethylene oxide (EO) to propylene oxide $(\mathrm{PO})$. 

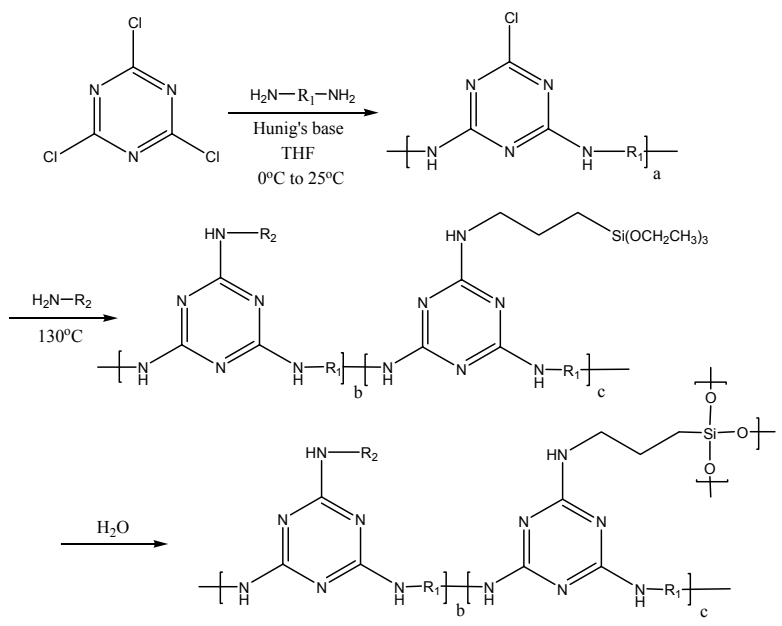

$\mathrm{R}_{1}=$ XTJ-500, XTJ-502

$\mathrm{R}_{2}=\mathrm{XTJ}-506, \mathrm{XTJ}-234, \mathrm{H}_{2} \mathrm{~N}$

Scheme 3. Polymer synthesis and cross-linking reaction. 


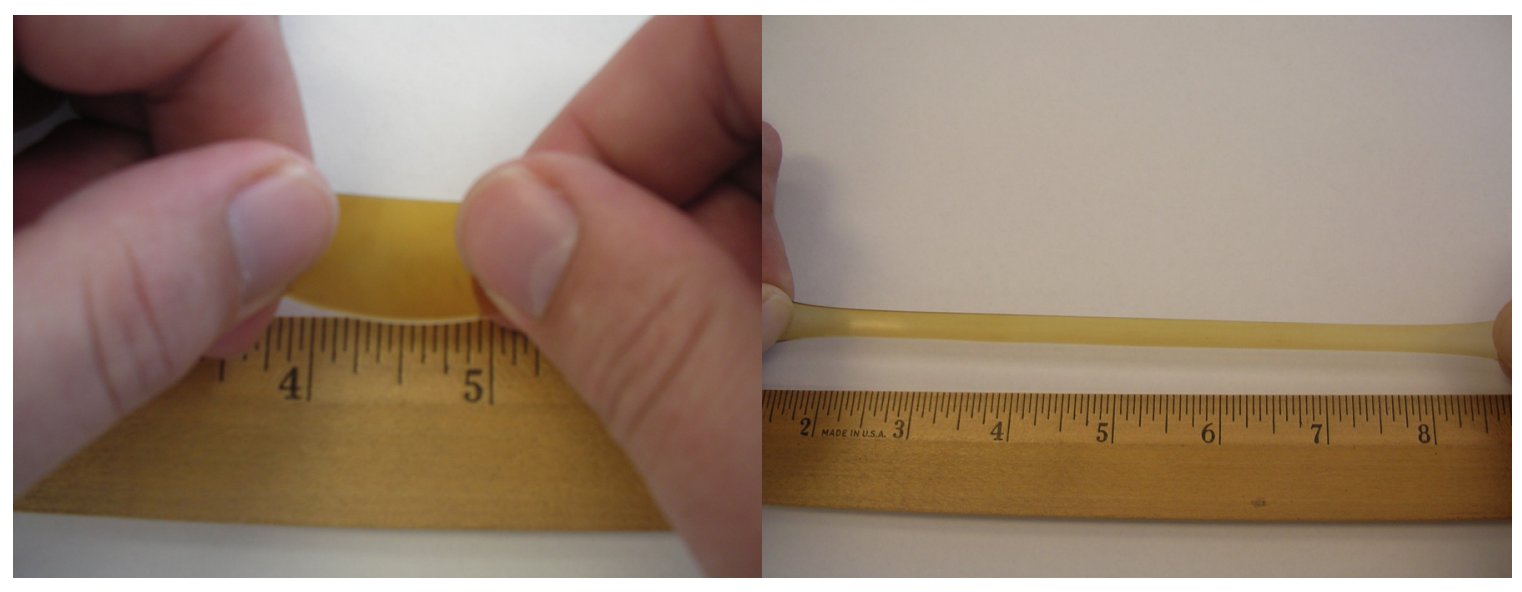

Figure 1. Polymer entry 42 before and after stretching. 


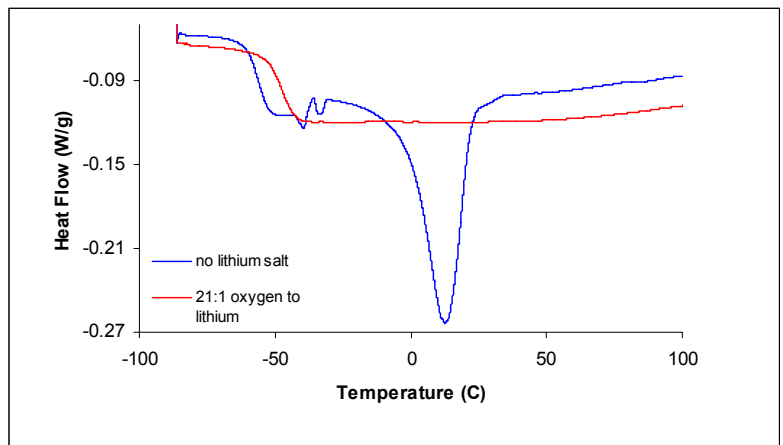

Figure 2. DSC analysis of polymer entries 16 and 19. Entry 16 contains lithium salt while entry 19 does not. 


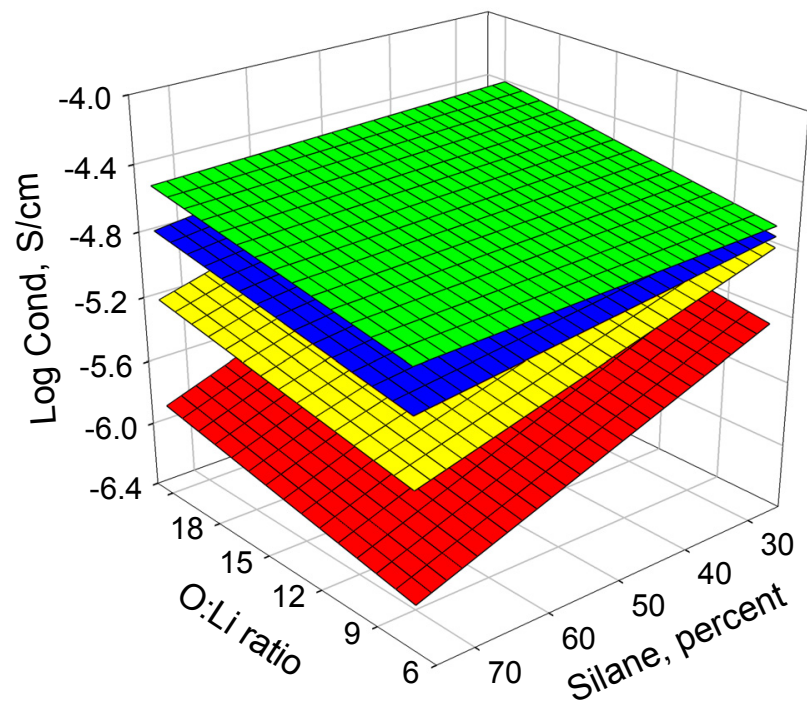

$\square$ Diamine=2000; monoamine $=2620$

Diamine $=2000$; monoamine $=1000$

$\square$ Diamine $=600$; monoamine $=2620$

Diamine=600; monoamine $=1000$

Figure 3. Response surface model predicting conductivities for 4 series of polymers. 


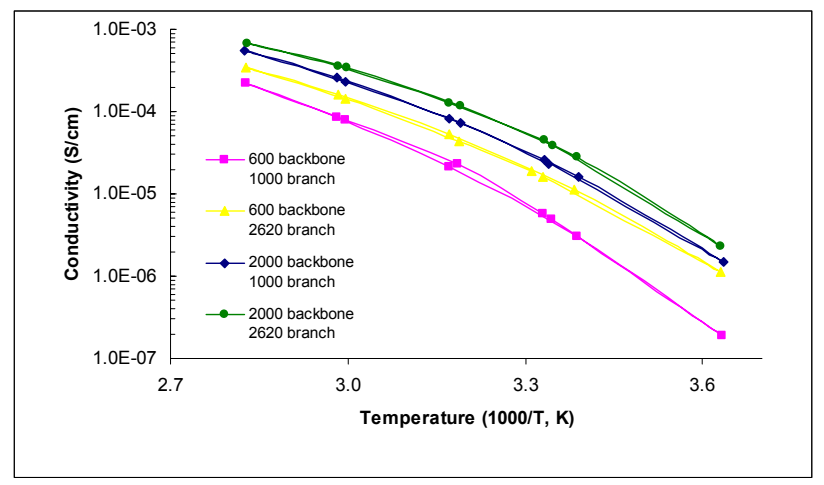

Figure 4. Temperature dependence of ionic conductivities for a series of polymers in which the branching site contains 50\% APTES and 50\% monoamino Jeffamine. The oxygen to lithium ratio is $21: 1$ in each case.
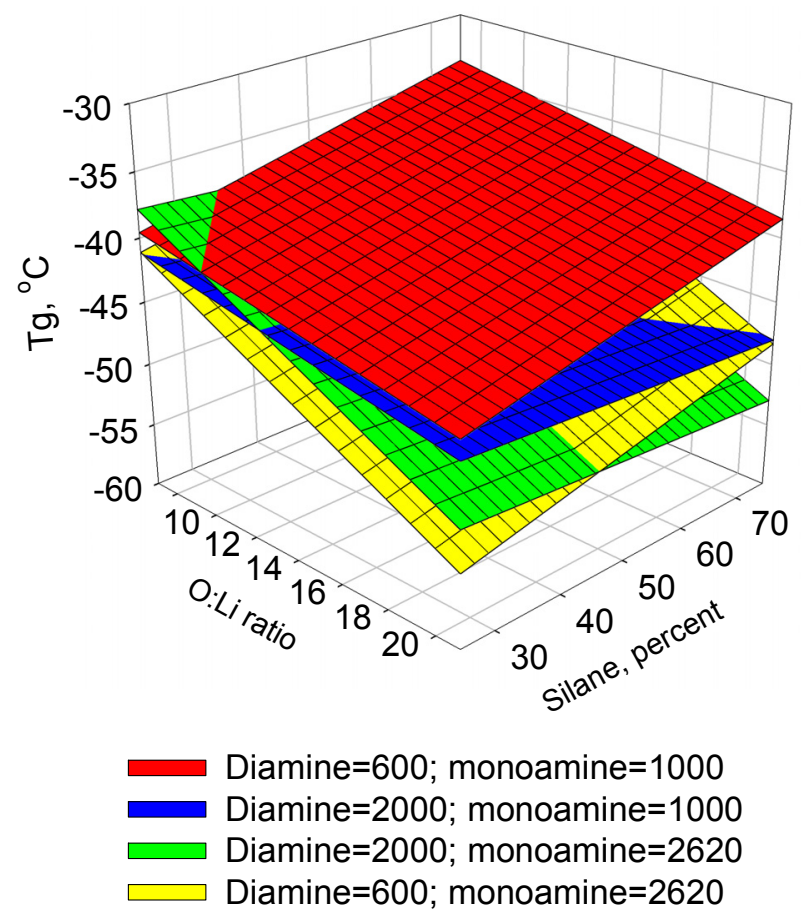

Figure 5. Response surface model predicting glass transition temperatures for 4 series of polymers. Note that the values of the percent silane are reversed compared to Figure 3 to provide the best viewing angle for all four surfaces. 


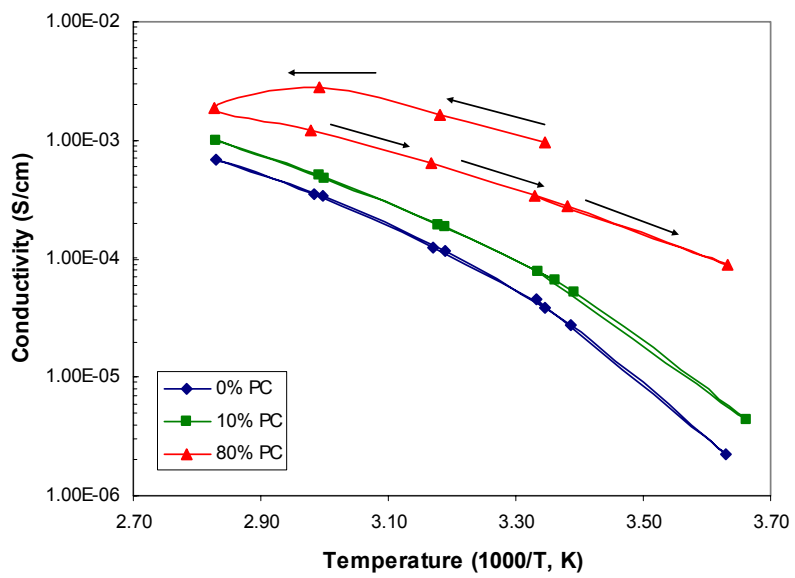

Figure 6. Temperature dependence of ionic conductivities for polymer 40 with propylene carbonate added. For the sample that contains $80 \%$ PC, the arrows indicate the increase, then decrease in measurement temperature. 